\title{
EL CONOCIMIENTO COMO SISTEMA EN EL TRATADO DE LA NATURALEZA DE DAVID HUME
}

\author{
Jean P. Martínez Zepeda ${ }^{1}$ \\ Pontificia Universidad Católica de Valparaíso \\ jean.martinez.z@mail.pucv.cl
}

\begin{abstract}
RESUMEN / ABSTRACT
La comprensión del conocimiento como sistema en el Tratado de la naturaleza humana de David Hume reconoce tres aspectos: primero, el conocimiento implica su distancia de la idea de sustancia y de ideas generales abstractas. Segundo, el conocimiento comprende la conexión entre impresiones e ideas. El enlace de nuestras impresiones e ideas surge del principio de asociación el cual ordena y reconfigura el conocimiento en virtud de la atracción, conexión articulada por las facultades de la memoria y la imaginación. Tercero, el conocimiento constituye un sistema constituido por la reiteración de hábitos y costumbres configurados por el principio de asociación desde la relación integrada de impresiones e ideas.
\end{abstract}

Palabras Clave: sistema, fuerza, relación, ideas, impresiones, sustancia.

KNoWLEDGE aS SYSTEM IN DAVID HUME's A TREATISE OF HuMaN NATURE

In A Treatise of Human Nature, David Hume's understanding knowledge as a system identifies three aspects: firstly, knowledge implies moving away from the idea of substance and abstract general ideas. Secondly, knowledge comprises the connection between impressions and ideas. The relation between our impressions and ideas arises from the principle of association which orders and reconfigures knowledge by virtue of the attraction, which is a connection articulated by the faculties of memory and imagination. Thirdly, knowledge is a system consisting of the repetition of habits and costumes configured by the principle of association from the integrated relation of impressions and ideas.

KEYWORDS: System, force, relation, ideas, impressions, substance.

$\overline{R A}$ Al final del camino empirista las tesis fundamentales relacionadas con la naturaleza del conocimiento en la filosofía inglesa llegan a su máxima expresión con el filósofo escocés David Hume (1711-1776). El pensamiento del filósofo de Edimburgo establecerá un nuevo horizonte que abrirá campo a reflexiones

1 Doctor en Filosofía, Pontificia Universidad Católica de Valparaíso. Dedicado en agradecimiento a Marcela Martínez, mi esposa. 
que avanzarán progresivamente a posiciones más complejas, sintetizadas en la visión del conocimiento humano como sistema. Sin embargo, si bien el camino planteado por Hume en su Tratado de la naturaleza humana ${ }^{2}$ (1739-1740) no será reconocido por la academia de su tiempo, hoy se erige como un referente al momento de analizar el concepto de conocimiento entendido desde una relación integrada entre impresiones e ideas, perspectiva que propone el rol de sensaciones, pasiones, emociones e ideas como aspectos determinantes en la configuración de una teoría general del conocimiento. En consideración a lo anterior, el objetivo del presente trabajo consiste en reconocer y analizar el concepto de conocimiento como sistema en el Tratado de la naturaleza humana de David Hume. El estudio, a continuación, propone destacar el concepto de conocimiento de Hume en términos de sistema, perspectiva que posibilita la comprensión de las operaciones del entendimiento en el Tratado.

Dentro del contexto del pensamiento empirista el presente trabajo formula tres interrogantes elementales: ¿Qué comprenden por ideas abstractas John Locke y George Berkeley?, ¿qué elementos configuran el origen de las ideas en Hume? y ¿por qué el conocimiento para Hume consiste en un sistema? Las respuestas a estas interrogantes suponen las siguientes hipótesis: primero, la comprensión de las ideas, para George Berkeley, no implica la existencia del concepto de sustancia, tal como lo afirma su predecesor John Locke, ni la existencia de ideas generales abstractas. La comprensión de las ideas surge del examen del lenguaje al establecer que los términos corresponden a determinadas ideas particulares, las cuales se transforman en ideas generales en la medida en que hacen referencia a conjuntos de ideas particulares. Segundo, la percepción humana en Hume comprende la conexión entre impresiones e ideas, donde las ideas son consideradas como copias débiles de las impresiones, las cuales difieren en su grado de fuerza y vivacidad. Las impresiones e ideas enlazadas a través del principio de asociación posibilitan el despliegue del conocimiento humano a cargo de la actividad de las facultades de la memoria y la imaginación. De este modo, el conocimiento no conlleva el examen de sustancias por descubrir ni la construcción de un conocimiento objetivo que descansa en la elucubración de razonamientos abstractos. Tercero, el conocimiento corresponde a un sistema abierto e integrado de hábitos y costumbres constituido por el principio de asociación mediante la conexión entre impresiones e ideas, pensamientos y emociones, delimitadas por las experiencias del sujeto. En atención a las temáticas expuestas y desde el análisis de las fuentes, el presente trabajo será desarrollado en tres etapas: primero, las ideas abstractas en John Locke y George Berkeley. Segundo, el origen de las ideas en David Hume. Tercero, el conocimiento como sistema en Hume.

2 En adelante la abreviatura y numeración de $A$ Treatise of Human Nature seguirá el orden convencional para la obra de Hume señalada con la letra (T.) y la correspondiente numeración de libro, capítulo, sección, párrafo y número de la edición de Selby-Bigge precedido de la abreviatura SB de acuerdo a la edición de David Fate Norton and Mary J. Norton (2000). El presente trabajo utiliza, además, la edición traducida al español por el profesor Félix Duque (2011) con la abreviatura D. para señalar la numeración de la página. 


\section{Las ideas abstractas en John Locke y George Berkeley}

El presente apartado tiene por propósito analizar las ideas abstractas desde las reflexiones de John Locke y George Berkeley, paso fundamental que nos permitirá su posterior comprensión en el pensamiento de David Hume. La tarea será dividida en dos partes: primero, análisis de las ideas abstractas en el Ensayo sobre el entendimiento humano ${ }^{3}$ (1690) del filósofo inglés John Locke y, segundo, análisis y crítica de las ideas abstractas en los Principios del conocimiento humano ${ }^{4}(1710)$ del pensador irlandés George Berkeley.

En primer lugar, en relación con el análisis de las ideas según John Locke. El filósofo inglés inicia su análisis de las ideas abstractas señalando que el ser humano tiene la capacidad natural de emitir sonidos, los cuales son comprendidos como signos de ideas ${ }^{5}$, esto es, signos de concepciones internas que pueden ser comunicados a otros (Locke 1994, p. 391). Para el autor, el análisis de nuestro conocimiento implica el detenido examen de palabras, en particular, de aquellas palabras generales que suponen la existencia de ciertas ideas abstractas, concebidas como esencias de ciertas clases de cosas (Locke 1994, p. 404). En consecuencia, la comprensión de las ideas abstractas implica el análisis del concepto de esencia, análisis que Locke desarrolla desde su distinción entre esencias reales y esencias nominales.

Primero, acerca de las esencias reales (real essences). Por esencias reales, el autor inglés comprende el ser de las cosas que, si bien es conforme a su propia constitución interna (Locke 1994, p. 433), nos es desconocido: "Y así la real e interna, pero generalmente en las sustancias, desconocida constitución de las cosas, de que dependen sus cualidades descubribles, puede llamarse su esencia" (Locke 1994, p. 406). El filósofo es claro en sostener la incapacidad de la mente humana para indagar la íntima constitución de las sustancias (Locke 1969, p. 234; Gibson 1968, pp. 92-93) de las cuales surgen, según su parecer, las distintas cualidades sensibles (Locke 1994, pp. 407-408, 435) y que clasifica, además, en dos tipos: las cualidades primarias y secundarias.

Por un lado, las cualidades primarias (primary qualities), inseparables de todos los cuerpos, se encuentran presentes en las partículas de la materia (particle of matter) y abarcan las ideas simples de solidez, extensión, forma, reposo, movimiento y número, modelos que existen en los cuerpos (Locke 1994, pp. 113-115). Por otro lado, las cualidades secundarias (secundary qualities), surgen en la mente humana

3 El presente estudio utiliza la traducción al español de An Essay Concerning Human Understanding por Edmundo O'Gorman (1994). En algunos casos se hace referencia a la edición de A. S. Pringle-Pattison (1969).

4 Se utiliza la traducción al español de A Treatise Concerning the Principles of Human Knowledge por Pablo Masa (1980). En algunos casos se hace referencia a la edición de G. J. Warnock (1967).

5 Un trabajo fundamental que permite una mirada integral respecto al análisis de las ideas y el significado de los términos en Locke es desarrollado por la profesora Giannina Burlando, sobre el mismo, véase Burlando 2013, pp. 132 y ss. 
desde las cualidades primarias, tales como las sensaciones, colores, sonidos, olores y gustos. La existencia de las cualidades secundarias obedece a la afección de los órganos sensibles por las partículas de solidez, extensión, forma, reposo, etc. (Locke 1994, p. 115). Por consiguiente, el ser humano desde el análisis y clasificación de sus cualidades sensibles puede distinguir diversas especies, las cuales reúne en clases de cosas y designa con nombres, signos que comprenden distintos individuos en conformidad con correspondientes ideas abstractas (Locke 1994, p. 455).

Segundo, respecto a las esencias nominales (nominal essences). Las esencias nominales corresponden a nombres generales producidos por la reunión de ideas simples en determinadas ideas complejas (Locke 1994, p. 442). Las esencias nominales, por tanto, suponen por las cosas como signos en cuanto representan a un conjunto de particulares (Locke 1994, p. 437). Para Locke, condiciones para la conformación de las esencias nominales serán, por un lado, que las ideas tengan una unidad a fin de poder establecer una idea única, y por otro, que las ideas particulares reunidas sean semejantes (Locke 1994, pp. 434, 447). Otro aspecto fundamental sobre las esencias nominales obedece a su inmutabilidad, la cual denota la existencia de ideas abstractas. Locke sostiene que todas las cosas se hallan sujetas al cambio, incluyendo la constitución interna de las cosas que reconocemos como esencias reales. No obstante, el significado de las esencias nominales tomadas en cuanto ideas abstractas en la mente, es decir, como especies de las cosas, se mantienen inmutables a pesar de todos los cambios a los que puedan ser sometidos los objetos (Locke 1994, p. 410).

En resumen, una idea abstracta constituye una determinada idea compleja que comprende un manojo de ideas simples reunidas bajo un nombre general, el cual es usado para comunicar nuestros pensamientos a otros (Locke 1994, pp. 396, 450). La razón de la formación de ideas generales procede más bien de la necesidad de la "eficacia" y "prontitud" en la comunicación mediante signos claros y definidos, que del examen de la naturaleza de las cosas (Locke 1994, p. 452). De este modo, la existencia de ideas abstractas se articula en función de palabras. En consecuencia, para el pensador inglés, las palabras generales representan a conjuntos de particulares que, en cuanto esencias nominales, son inmutables, a saber, poseen una "referencia" a algo invariable desprovista tanto de las circunstancias de cierto tiempo y lugar como de cualquier idea que pueda determinarla a una existencia particular (Locke 1994, p. 400), aspecto que será analizado por David Hume posteriormente.

En segundo lugar, sobre el análisis de las ideas abstractas según George Berkeley. Berkeley escribe los Principios del conocimiento humano con el propósito de esclarecer ciertos problemas fundamentales de la filosofía asociados a la incipiente lectura materialista del conocimiento en el pensamiento de su época. En la introducción a su obra el autor señala dos críticas, a saber, a la existencia de ideas abstractas allende la experiencia, y al concepto de sustancia. Ambas temáticas ya planteadas por John Locke.

En este contexto, Berkeley juzga la posición de su predecesor John Locke en torno al sentido de las ideas abstractas que procede de su distinción entre cualidades primarias y secundarias, en particular, al reconocer el origen de las ideas simples desde la existencia de cualidades primarias que surgen de los cuerpos en sí mismos. Según 
Berkeley, desde la posición de Locke, como la de distintos pensadores a lo largo de la historia, se señala la capacidad de la mente para generalizar ideas, ya sea al reconocer cualidades particulares desde las cosas como también para concebir aspectos comunes entre las distintas cualidades. De este modo, ambos elementos permitirían el origen de ideas generales abstractas (Berkeley 1980, pp. 31-34). Locke reconoce, además, que la capacidad del lenguaje denota la capacidad de abstracción de los seres humanos, situación que se confirma al identificar la estrecha relación entre las palabras generales como signos y las ideas generales abstractas: “(...) las palabras adquieren sentido general porque se convierten en signos de ideas generales (Ensayo sobre el entendimiento humano, Lib. III, cap. III, sec. 6)" (Berkeley 1980, p. 38).

Ahora bien, para el pensador irlandés, desde el análisis del lenguaje es posible inferir que las palabras solo adquieren el sentido de ideas generales como signos en tanto representan a un conjunto de ideas particulares y no de ideas generales abstractas: “(...) una palabra adquiere sentido general por convertirse en signo no de una idea general abstracta, sino de varias ideas particulares" (Berkeley 1980, p. 38). El origen de la falsa noción de ideas abstractas se encuentra en el mal uso del lenguaje. Una primera confusión surge al indagar en torno a una "precisa" y "determinada" significación de alguna idea, lo cual conduce a suponer su existencia. No obstante, según Berkeley: “(...) no hay tal significación precisa y determinada de cada palabra, pues todas ellas representan indiferentemente un gran número de ideas particulares" (Berkeley 1980, p. 47). Berkeley aclara que si bien es necesario conservar las definiciones de los términos, no lo es limitar el significado a una misma idea. Una segunda confusión proviene de sostener que el fin del lenguaje es exclusivamente la comunicación de ideas y que todo nombre significa una idea determinada; por el contrario, para Berkeley, el lenguaje posibilita la comunicación de los más diversos aspectos de la vida humana entre los cuales se incluye la expresión de pasiones, sentimientos o emociones (Berkeley 1980, pp. 49-50).

Cabe añadir que, para el filósofo irlandés, el significado de los términos implica, por un lado, que su universalidad no radica en una realidad absoluta y positiva sino en la relación que estos términos tienen con los particulares a los que representan y significan, razón por la cual las palabras particulares se vuelven universales (Berkeley 1980 , p. 43$)^{6}$. Por otro lado, los términos representan nuestras concepciones internas que son determinadas por el uso que damos a nuestras palabras, según el autor: "El uso regula la propiedad del lenguaje" (Berkeley 1980, p. 98).

En atención a lo expuesto anteriormente es posible establecer que la lectura nominalista de Berkeley implica su distanciamiento de las ideas generales abstractas, entre ellas la idea abstracta de "sustancia material" como garante de un conocimiento objetivo en el cual tendrían su origen ${ }^{7}$ nuestras ideas (Berkeley 1980, pp. 71, 125). De

Otra lectura se encuentra en Pringle-Pattison (Locke 1969, p. 352).

En términos generales, el denominado inmaterialismo de Berkeley se distancia de Locke respecto al origen de nuestras ideas simples. Mientras para el filósofo irlandés la causa de 
este modo, la posición de Berkeley supone que las ideas impresas en nuestra mente son reales, pero no desde la pretensión de su existencia como independientes al espíritu humano, sino en cuanto son percibidas por el propio entendimiento, según sus palabras: “(...) puesto que el ser de una sensación o idea está en que sea percibida" (Berkeley 1980, p. 129) ${ }^{8}$.

\section{El origen de las ideas según David Hume}

El presente apartado tiene por objeto examinar el origen de las ideas en la comprensión general del conocimiento en el Tratado de la naturaleza humana de David Hume. Para lograr este propósito el estudio en cuestión se articula en función de dos partes: primero, análisis sobre el origen de las ideas y, segundo, crítica al concepto de sustancia e ideas abstractas. El análisis sobre el origen de las ideas de acuerdo a Hume mantiene la argumentación planteada por George Berkeley en el sentido de que en ambas posiciones se destaca el distanciamiento de un análisis esencialista de las sustancias e ideas abstractas presentes en las reflexiones de John Locke. La posición crítica de Hume constituirá, por tanto, un aspecto crucial para el progreso de su concepto de conocimiento que germinará en su concepción como sistema.

En primer lugar, en torno al análisis del origen de las ideas. La posición de David Hume acerca de la naturaleza del conocimiento comprende el examen de la percepción, esto es, respecto al origen de las impresiones e ideas según lo expresado en su Tratado de la naturaleza humana. En su obra, Hume manifiesta la necesidad de una revisión del proceso intelectual desde el campo de la experiencia para un acabado análisis de los pensamientos, sentimientos y comportamientos humanos ${ }^{9}$, perspectiva inspirada

nuestras ideas es Dios, para Locke nuestras ideas provienen de la abstracción de la materia. Según Berkeley: “(...) Por otra parte, las cosas percibidas por el sentido se pueden decir externas con relación a su origen, en cuanto no son engendradas desde dentro de la mente, sino 1) impresas por un espíritu distinto de aquel que los percibe. Igualmente los objetos sensibles pueden decirse independientes de la mente en otro sentido, a saber, 2) cuando existen en algún otro espíritu. Y así, al cerrar yo los ojos, pueden continuar existiendo las cosas que yo vea, pero existirán en otra mente o espíritu" (Berkeley 1980, p. 129).

8 "(...) since the very being of a sensation or idea consists in being perceived" (Berkeley 1967, p. 110).

$9 \quad$ Hume perfecciona esta perspectiva en su obra posterior denominada An Enquiry concerning human understanding (1748). La edición utilizada en español corresponde a la traducción de V. Sanféliz y C. Ors (2004). Las abreviaturas para el Enquiry son: E. para libro, número para sección, parte y párrafo de acuerdo a la edición de T. L. Beuchamp y SO para indicar la numeración de la página de la traducción al español. Por otro lado, cabe señalar que la pretensión del examen de una ciencia del hombre ha de recoger el campo de la acción, el cual considera una mirada general del hombre que integre su dimensión intelectual y emotiva; en palabras del autor escocés: "Concedo tu pasión por la ciencia, les dice, pero haz que tu ciencia sea humana de tal modo que pueda referirse directamente a la acción y la sociedad" (E.1,6, SO.37). 
en el pensador de la época Francis Hutcheson y sus trabajos en torno a moral y estética (Santucci 1971, p. 11; Stroud 1986, pp. 23, 323). El análisis del conocimiento, según Hume, pretende llevar a cabo una teoría concordante con los descubrimientos de la ciencia de su tiempo, marco desde el cual establece que el propósito de su obra consiste en realizar un examen de las causas de las percepciones e ideas. Para conseguir este objetivo, en el libro primero del Tratado de la naturaleza humana, el pensador escocés plantea la necesidad de explorar la comprensión de los principios que determinan la vida intelectual; mientras, en el libro segundo, los de la vida afectiva, estudio que concierne al análisis de las pasiones, emociones y sentimientos. Ambos libros, según Noxon: “(...) constituyen en sí mismos una cadena completa de razonamientos” (Noxon 1987, pp. 21-22).

En términos generales, podemos aseverar que el conocimiento para Hume surge de la estricta relación entre impresiones (impressions) e ideas. La actividad de nuestro entendimiento se configura a partir de impresiones que comprenden sensaciones, pasiones y emociones, todas ellas caracterizadas principalmente por su fuerza (force) y vivacidad (vivacity). En este ámbito, Hume sostiene que desde las impresiones surgen otras imágenes más tenues denominada ideas, “copias de las impresiones” (T.1.1.1.1 SB1, D.43; E.2.1, SO.53). Por consiguiente, las diversas ideas en nuestro entendimiento deben su causa a sus correspondientes impresiones (Ayer 1988, p. 59). En palabras del autor:

Todas las percepciones de la mente humana se reducen a dos clases distintas, que denominaré impresiones e ideas. La diferencia entre ambas consiste en los grados de fuerza y vivacidad con que inciden sobre la mente y se abren camino en nuestro pensamiento o conciencia. A las percepciones que entran con mayor fuerza y violencia las podemos denominar impresiones; e incluyo bajo este nombre todas nuestras sensaciones, pasiones y emociones tal como hacen su primera aparición en el alma. Por ideas entiendo las imágenes débiles de las impresiones cuando pensamos y razonamos (...) (T.1.1.1.1 SB1, D.43).

La cuestión de la relación entre impresiones e ideas ${ }^{10}$ constituye el eje central al momento de comprender la tesis de Hume en torno a la génesis del conocimiento ${ }^{11}$,

10 Sobre esta relación, Stroud afirma: "Un poco de reflexión acerca del contenido de nuestra mente muestra que, en palabras de Hume, 'todas las percepciones de la mente son dobles, y aparecen como impresiones y como ideas' (pp. 2-3). Es decir, cuando examinamos lo que hay en nuestras mentes, al parecer hallamos que todos los contenidos vienen en parejas, y que la única diferencia entre los miembros de cada pareja es una diferencia en los grados de fuerza y vivacidad con que hiere la mente" (Stroud 1986, p. 36). Por otro lado, sobre el rol de los sentimientos en la cuestión de la relación entre impresiones e ideas, véase Santucci 1971, pp. 24-25.

11 Respecto al denominado principio de copia, Hume afirma: “(...) que cuando analizamos nuestros pensamientos o ideas, no importa lo complejos o sublimes que sean, siempre encontramos que se resuelven en ciertas ideas simples copiadas de un sentimiento o sensación precedente" (E.2.5; SO59). Sobre este principio de copia como proceso intelectual, Noxon afirma: "Desde mi punto de vista el principio de copia no es ni una 'generalización empírica' 
según el autor escocés: "De esta conjunción constante de percepciones semejantes concluyo inmediatamente que existe una gran conexión entre nuestras impresiones correspondientes y nuestras ideas, y que la existencia de las unas tiene una influencia considerable sobre la de las otras" (T.1.1.1.8 SB5, D.47). En este contexto, Noxon señala que el denominado principio de copia pretende generar claridad respecto a la comprensión filosófica del origen de las ideas evitando la confusión sobre sus causas, en particular, al favorecer la comprensión del proceso que determina el enlace y configuración del conocimiento (Noxon 1987, p. 143).

Hume asevera que el origen de impresiones e ideas en la mente procede de la acción de facultades distintas, puesto que, mientras en algunas ocasiones las ideas aparecen con mayor vivacidad por el rol de la memoria, en otras son más débiles y surgen de la actividad de la imaginación: "Ya a primera vista es evidente que las ideas de la memoria son mucho más vívidas y fuertes que las de la imaginación, y que la primera facultad colorea sus objetos con mayor precisión que la segunda" (T.1.1.3.1 SB9 D.52). El pensador escocés incluye en su análisis la distinción de ideas, la cual concibe en dos tipos: las ideas simples y complejas. Las ideas simples son unidades que no poseen división, caso contrario a las complejas, las cuales representan las ideas que pueden ser analizadas en sus componentes más simples, distinción que se extiende también a la clasificación de las impresiones (T.2.1.1.2 SB275 D.387-388). El mismo Hume presenta como ejemplo de ideas simples aquellas que representan el color, sabor y olor que corresponden a una determinada idea compleja como, por ejemplo, la idea de una manzana (T.1.1.1.2 SB2, D.44). Respecto al origen de las ideas, el pensador de Edimburgo atribuye a la facultad de la imaginación el rol de la formulación de las ideas complejas, ya sea mediante combinaciones en forma arbitraria de ideas simples, dando con ello lugar a toda clase de quimeras (como cuando pensamos un ave como el Fénix) o de asociaciones determinadas por el denominado principio de asociación o atracción (principle of association or attraction), axioma desde el cual el pensador explica la atracción generada bajo ciertos principios, que el autor expresa en tres leyes fundamentales: 1) criterio de semejanza (resemblance), 2) contigüidad espacio y tiempo (contiguity in time or place) y, finalmente, 3 ) causa y efecto (cause and effect) (T.1.1.4.1 SB11, D.55; E. 3.2, SO67 ).

De este modo, el autor del Tratado utiliza el principio de asociación $n^{12}$ para describir la atracción de ideas en la mente desarrollada por la facultad de la imaginación, atracción que da origen a un sinnúmero de combinaciones y relaciones posibles, conexiones desplegadas en virtud de las propias experiencias del sujeto. En palabras del escocés: "Hay aquí una especie de atracción, que se encontrará tiene en el mundo

ni una 'tautología pretenciosa' (...) El principio de copia es una regla de procedimiento: Prescribe una técnica para investigar los términos sospechosos de carecer del significado que se les atribuye en las teorías filosóficas" (Noxon 1987, p. 142).

12 Una explicación sintética en torno a la "conexión de ideas" en la historia del pensamiento es mostrada por Pringle-Pattison en (Locke 1969, p. 217). Finalmente, otra lectura sobre este principio en los tiempos modernos es realizada por Russell (Russell 1992, p. 111). 
mental efectos tan extraordinarios como en el natural, y que se revela en formas tan múltiples como variadas" (T.1.1.4.6 SB13, D.57). El pensador de Edimburgo, al intentar describir esta conexión la califica como una "fuerza suave" (gentle force) (T.1.1.4.1 SB10, D.54) que enlaza según su libertad las impresiones e ideas ${ }^{13}$, posición que nos permite visualizar la plasticidad que posee, para Hume, el conocimiento humano al reconocer el rol de la "actividad" del mismo cerebro que por la imaginación puede conectar o desconectar a voluntad las ideas:

Observaré, por tanto, que como la mente tiene la facultad (power) de suscitar cualquier idea que le plazca siempre que envía a los espíritus a la región del cerebro en que está situada esa idea, dichos espíritus suscitan la idea cuando corren justamente por las huellas adecuadas, agitando la célula que corresponde a la idea (T.1.2.5.20 SB61, D.116).

Además, otra lectura del conocimiento desde una perspectiva psicológica reconoce que el dinamismo del razonamiento humano gravita en atención a la comparación de objetos con el fin de identificar sus distintas relaciones, a diferencia de la recepción pasiva de las impresiones por los sentidos (T.1.3.2.2 SB73, D.132). La mente posee la capacidad de identificar una determinada percepción rompiendo sus relaciones con el objeto de analizar con detención un aspecto particular (T.1.4.2.39 SB207, D.300) y, también, el poder de articular impresiones e ideas por la facultad de la imaginación. Desde este enfoque, nuestras percepciones constituyen los objetos del pensamiento, razón por la cual Hume sostiene la imposibilidad de ir más allá de los mismos. En palabras del filósofo escocés: "La imaginación sigue naturalmente esta serie de pensamientos: nuestras percepciones son nuestros únicos objetos" (T.1.4.2.50 SB213, D.308). El autor del Tratado, finalmente, junto con exponer la labor del principio de asociación, sostiene dos aspectos fundamentales que permiten la unidad y progreso de nuestro conocimiento; estos son: la constancia de nuestras percepciones, al atribuir a los objetos una existencia continua que acompañada, además, de una determinada coherencia, mantiene la unidad de nuestra percepción a pesar de los posibles cambios en los objetos conocidos. La unidad de la constancia y coherencia de nuestras percepciones posibilita, por tanto, la construcción de nuestro conocimiento empírico (T.1.4.2.20, SB195, D.286) ${ }^{14}$.

Cabe destacar que, para el filósofo de Edimburgo, la configuración continua de combinaciones de ideas a partir del principio de asociación permite una comprensión del mundo interior a partir de una dinámica de fuerzas semejantes a las plasmadas en

13 Según Hume: "Entre los efectos de esta unión o asociación de ideas no existe ninguno tan notable como las ideas complejas, que son normalmente el objeto de nuestros pensamientos y razonamientos y que surgen por lo general de un principio de unión entre nuestras ideas simples" (T.1.1.4.7 SB13, D.57). Sobre el principio de asociación en las impresiones, véase T. 2.1.4.3 SB283, D.399.

14 Véase también Santucci 1971, p. 60; Ayer 1988, p. 77. 
los trabajos de Newton sobre la gravedad ${ }^{15}$ en su explicación de la física. Es posible interpretar, desde este punto, que el impulso por desarrollar una teoría científica del conocimiento humano pudo provocar en el pensador escocés una posible vía para configurar su teoría de la atracción de ideas al momento de desarrollar un discurso que fuese concordante con el conocimiento académico de la época (Noxon 1987, p. 123; Stroud 1986, pp. 14-15, 21).

Por otro lado, el pensador escocés establece la capacidad de la mente humana de incrementar sus conocimientos con el objeto de construir en forma progresiva un sinnúmero de experiencias que determinan nuestra comprensión de los hechos y donde la memoria tiene un rol capital; en palabras de Hume: "Los objetos externos se ven y se tocan, y se manifiestan a la mente; esto es, adquieren relación con un montón conectado de percepciones, que influyen muy notablemente sobre éstas al aumentar su número mediante reflexiones y pasiones presentes, abasteciendo a la memoria" (T.1.4.2.40 SB207, D.301). Ahora bien, para el autor del Tratado, el análisis de la experiencia adquirida se puede realizar desde el fundamental análisis del lenguaje, es decir, desde el examen de las proposiciones que Hume clasifica en dos clases: relaciones de ideas (relations of ideas) y cuestiones de hecho (matters of fact) ${ }^{16}$ (Ayer 1988, p. 61; Stroud 1986, pp. 20, 233-234). Respecto a la distinción, el autor escocés afirma: "Todos los objetos que caen bajo la razón o la investigación humana pueden dividirse de forma natural en dos clases, a saber: relaciones de ideas y cuestiones de hecho" (E.4.1.1, SO.85). Las relaciones de ideas, consisten en proposiciones analíticas relativas a ciencias tales como la geometría, álgebra y la aritmética, afirmaciones intuitiva y demostrativamente ciertas que pueden ser deducidas sin directo concurso de la existencias de objetos; las cuestiones de hecho, en cambio, serán proposiciones sintéticas que pueden ser concebidas por la mente en cuanto concordantes con los hechos, esto es, sin contradicción a lo dictado por la propia experiencia.

En definitiva, desde lo antes planteado es posible afirmar que, para el pensador de Edimburgo, la actividad de la memoria e imaginación bajo los principios de copia y asociación permitirá la articulación de ideas complejas que, enlazadas bajo los principios mencionados, darán lugar a la interpretación del conocimiento como un

15 La formulación de hipótesis experimentales y no especulativas posibilita el análisis y desarrollo del conocimiento, posiciones en las que Hume y Newton coinciden, pero de las cuales también difieren (Noxon 1987, p. 95).

16 Según Pringle-Pattison estos tipos de proposiciones tienen un antecedente en el pensamiento de Locke (Locke 1969, p. 258). Cabe añadir que acerca de las cuestiones de hecho, el filósofo de Edimburgo señala su fundamentación en la relación causa y efecto. Las afirmaciones relativas a cuestiones de hecho se conforman en virtud de la percepción de causas y efectos semejantes; en palabras del autor: "De causas que parecen similares esperamos similares efectos" (E.4.2.20; SO.105). La semejanza en materia del examen de los hechos nos conduce a reconocer la conformación del conocimiento desde la costumbre, aspecto central en el desarrollo de la experiencia. También es posible descubrir en Locke una potencial lectura sobre el rol de la costumbre en materia del conocimiento (Locke 1969, p. 218). 
sistema. De esta manera, la estructura constituida por la articulación o relación entre impresiones e ideas ${ }^{17}$, y capaz de ser analizada a partir de proposiciones, será considerada por el filósofo escocés como realidad, perspectiva que afirma el rol del conocimiento en la configuración de nuestro mundo (T.1.3.9.3 SB108, D.177). La concepción del conocimiento como sistema será abordada en el siguiente apartado.

En segundo lugar, en torno a la crítica al concepto de sustancia e ideas abstractas. Primero, sobre la crítica a la idea de sustancia como subyacente a las cosas. La comprensión de la idea de sustancia en Hume continúa el análisis de Berkeley respecto a la crítica a una idea de sustancia subyacente a las cosas, tal como fue elaborada por John Locke ${ }^{18}$ (T.1.1.6.2 SB16, D.61). Para el filósofo de Edimburgo, la idea de sustancia no es producto de las impresiones sensibles ni de las impresiones de reflexión (únicos elementos que nos disponen el desarrollo de ideas), sino más bien surgen del rol de la imaginación que atribuye a un nombre una colección de ideas simples: a este término, en general, se denomina "sustancia" (T.1.1.6.2 SB16-17, D.61-62). De esta manera, solo es posible concebir "nominalmente" la existencia de la idea de sustancia, esto es, al atribuir a un nombre una colección de determinadas cualidades particulares. En palabras del autor: "La idea de sustancia, como la de modo, no es sino una colección de ideas simples unidas por la imaginación y que poseen un nombre particular asignado a ellas, mediante el cual somos capaces de recordar -a nosotros y a otros-esa colección" (T.1.1.6.2 SB16, D.61).

Segundo, sobre la crítica a las ideas abstractas. En relación con las ideas abstractas, el pensador escocés asume el análisis planteado por Berkeley (Ayer 1988, p. 59) en torno a la naturaleza de los términos: "Un gran filósofo ha combatido la opinión tradicional sobre este asunto, afirmando que todas las ideas generales no son sino ideas particulares añadidas a un cierto término que les confiere mayor extensión, y que hace que recuerden ocasionalmente a otros individuos similares a ellas" (T.1.1.7.1 SB17, D.62). Es necesario reconocer, por tanto, que el grado de significación de los términos para Hume no radica en la referencia a sustancias o entidades abstractas, incomprensibles y

17 Un esquema que puede facilitar al lector la comprensión de la posición de Hume respecto al origen de las ideas es formulado por el profesor Stroud del siguiente modo:

1. No hay pensamiento o actividad mental a menos que haya una percepción ante la mente.

2. Toda percepción es o una impresión o una idea.

3. Toda percepción es o simple o compleja.

4. Toda percepción compleja está íntegramente constituida por percepciones simples.

5. Para toda idea simple hay una impresión simple correspondiente.

6. Toda idea simple surge en la mente como efecto de su impresión simple correspondiente.

7. No hay impresiones de reflexión sin alguna impresión de sensación. Por lo tanto,

8. No hay pensamiento o actividad mental a menos que haya impresiones de sensación (Stroud 1986, p. 40).

18 Sobre las distinciones entre las ideas en Hume y Locke, Stroud 1986, p. 35; Gibson 1968, pp. 94-95; Burlando 2013, p. 122. 
distantes de nuestras impresiones ${ }^{19}$, sino en la "referencia" a "conjuntos de particulares" y al "uso" que damos a los mismos términos (Ayer 1988, p. 59). Por otro lado, Hume asume que el problema fundamental en la errada comprensión de las ideas abstractas se encuentra en pretender su existencia prescindiendo justamente de determinaciones particulares, posición ya justificada por Locke ${ }^{20}$ (Locke 1994, p. 400). Para el pensador de Edimburgo, por el contrario, las impresiones contienen grados de cantidad y cualidad, del mismo modo que las ideas, sus respectivas copias que se hallan limitadas a estos dos aspectos. (T.1.1.7.5 SB19, D.65). En definitiva, es posible afirmar que la crítica al concepto de sustancia e ideas abstractas permite al autor escocés establecer los reales alcances de nuestras percepciones y dar un paso crucial que permitirá la proyección de una nueva lectura del conocimiento contenido bajo la visión de un sistema, cuestión que será analizada a continuación.

\section{El conocimiento como sistema en David Hume}

La filosofía empirista de Hume se distancia considerablemente de las filosofías precedentes, en particular, al plantear una nueva perspectiva respecto al origen de las ideas y del estatuto del conocimiento. Se ha insistido en que el pensamiento de David Hume se plasma en términos del alejamiento de la idea de sustancia ${ }^{21}$ y de las ideas generales abstractas como garantes de las ideas de sensación, tal como acontece en el análisis de John Locke. En consideración de lo anterior, el objetivo del presente apartado consiste en reconocer el concepto de conocimiento como sistema en el Tratado de la naturaleza humana. Para cumplir este objetivo el presente apartado se articula en función del examen de tres temas centrales: primero, el concepto de conocimiento como hábito y costumbre, segundo, el conocimiento como sistema y, tercero, el conocimiento como sistema integrado de pensamientos y emociones.

Primero, el conocimiento como colección de hábitos y costumbres. La configuración de los procesos mentales determinados por la costumbre ${ }^{22}$ se constituye por la reiteración

19 Véase la crítica de Russell a Hume en Russell 2009, p. 712.

20 Sobre puntos de encuentro y diferencias entre las ideas de Locke y las percepciones en Hume, véase Burlando 2013, p. 122.

$21 \quad$ Véase una lectura de la idea de sustancia en Russell 2009, p. 713.

22 Respecto a la costumbre, Hume afirma: "Este principio es la costumbre o el hábito. Pues dondequiera que la repetición de cualquier particular acto u operación produce una propensión a renovar este mismo acto u operación, sin que seamos impelidos a ello por ningún razonamiento o proceso del entendimiento, decimos que esta propensión es el efecto de la costumbre" (E.5.1.5, SO119). Hume continúa su argumentación definiendo el importante rol de la costumbre al momento de analizar la configuración de nuestro conocimiento: "La costumbre, pues, es la gran guía de la vida humana. Es este principio solo el que nos torna útil la experiencia, y nos hace esperar para el futuro un curso de eventos similar al que se ha dado en el pasado. Sin la influencia de la costumbre seríamos completamente ignorantes de toda cuestión de hecho que fuera más allá de lo inmediatamente presente a los sentidos 
de actos mentales, los cuales son denominados hábitos ${ }^{23}$. Los hábitos conforman la base de nuestras experiencias y se erigen a partir de la regularidad de nuestras impresiones e ideas mediante los cuales podemos incluso establecer posibles condiciones futuras de ciertos hechos. Sin embargo, la reiteración de actos mentales también puede ser fuente de error cuando el conocimiento no se sostiene a partir de una experiencia fundada en los hechos, es decir, basada directamente en las impresiones (T.1.3.8.14 SB105, D.173), lo cual mueve la imaginación a la creencia ${ }^{24}$. Para Hume es evidente que las creencias no existen en el orden de las ideas sino más bien en el modo en que son concebidas o las siente la mente (E.5.2.12, SO.131), esto es, no se articulan en relación con los hechos. Por consiguiente, el sentido de hábito en el pensamiento de Hume corresponde solo a un determinado conocimiento que se sostiene en el campo de la experiencia; en el caso contrario, los hábitos pueden ser fuente de confusión por el excesivo rol de la imaginación. En este contexto, es necesario señalar que la adquisición de hábitos es causada por la reiterada percepción de sucesivos efectos frente a determinadas causas (T.1.3.8.14 SB105, D.173-174).

Ahora bien, el filósofo de Edimburgo propone el fundamental examen del lenguaje al momento de analizar determinados hábitos y costumbres articulados desde la relación entre ideas y palabras. Para el filósofo escocés, según lo ya expuesto, las ideas (incluso las abstractas) son individuales; sin embargo, el significado de las mismas se construye en virtud de las semejanzas que admitimos de distintos objetos a los cuales aplicamos el mismo nombre (T.1.1.7.6 SB20, D.66). Ahora bien, podemos destacar que la posición de Hume inaugura una perspectiva revolucionaria para su época ${ }^{25}$ al instalar un nuevo enfoque al momento de examinar las ideas complejas; específicamente desde el análisis de los "significados" y "referencias" de los términos circunscritos, tanto a

o a la memoria" (E.5.1.6, SO.123). Por otro lado, en torno a la idea de conexión necesaria entre causa y efecto, véase E.7.2.30, SO.185.

23 Según Guillermo de Ockham, filósofo franciscano inglés del siglo XIV, los conocimientos son concebidos como cualidades del alma, esto es, como ciertos hábitos. Ockham 1986, p. 51 .

24 Según Hume: "Y en la filosofía no podemos ir más allá de aseverar que la creencia es algo sentido por la mente que distingue las ideas del juicio de las ficciones de la imaginación. Les da más peso e influencia, las hace parecer de mayor importancia, las refuerza en la mente, y las torna principios gobernantes de nuestras acciones" (E.5.2.12, SO131). Noxon atribuye a John Stewart el juicio de que el propósito central del primer libro del Treatise es mostrar el rol de la creencia (Noxon 1987, p. 153).

25 Una interpretación respecto a la aspiración de Hume con el principio de asociación y su real impacto en el pensamiento de su época es expresado por Noxon del siguiente modo: "Cuando (Hume) escribió el Treatise, deseaba derivar la pasión y la acción, el amor, el odio, la compasión, la benevolencia, la aversión, la malicia, el resentimiento y el sentido de la justicia, obligación, ley y corrección a partir de las fuerzas elementales de placer y dolor, junto con el principio de asociación, concebidos en analogía con los axiomas del movimiento y la fuerza de la gravedad. Al parecer, de este ejercicio esforzado y frustrante sacó la convicción de que los tiempos aún no estaban maduros para realizar una síntesis newtoniana en las ciencias humanas" (Noxon 1987, p. 122). 
la experiencia del sujeto como al uso común de los términos, aspectos que inciden en el desarrollo de las distintos hábitos y costumbres.

Una explicación de la operatoria en torno a la relación entre ideas, palabras y hábitos es expuesta en los siguientes términos: "La palabra despierta una idea individual, a la vez una cierta costumbre, y tal costumbre produce cualquier otra idea individual que podamos tener la ocasión de emplear" (T.1.1.7.7 SB21, D.67). De esta manera, las ideas particulares, que adquirimos mediante la experiencia, y que se sostienen desde las impresiones e ideas que tenemos de las mismas, pueden ser consideradas en relación con términos ${ }^{26}$ generales; según Hume: “(...) Una idea particular se convierte en general al ser unida a un término; esto es, a un término que por conjunción debida a la costumbre guarda relación con muchas otras ideas particulares y las hace fácilmente presentes a la imaginación" (T.1.1.7.10 SB22, D.69). Las ideas particulares, por lo tanto, se vuelven generales por el hábito o costumbre de representar un conjunto de singulares, en ello descansa el significado de los términos generales; en palabras del autor: "Si las ideas son particulares en naturaleza y al mismo tiempo finitas en su número, solo por costumbre pueden hacerse generales en su representación y contener bajo sí un número infinito de otras ideas" (T.1.1.7.16, SB24, D.71).

Segundo, el conocimiento como sistema. En el ámbito del conocimiento, esto es, del estricto contenido de la mente, podemos reconocer que las ideas se enlazan en conjuntos de grupos denominados sistemas configurados por el entendimiento. La mente, por tanto, tiene la habilidad de clasificar y articular en forma constante nuevas ideas complejas por el poder de la misma memoria e imaginación asociados al conocimiento por costumbre. De este modo, el análisis del conocimiento para Hume implica el examen de las impresiones e ideas como su conexión. En palabras del autor:

Es evidente que cuando algo presente a la memoria excita la mente con una vivacidad tal que lo asemeja a una impresión inmediata, deberá convertirse en punto importante en todas las operaciones de la mente y distinguirse con facilidad de las meras ficciones de la imaginación. De estas impresiones o ideas de la memoria formamos una especie de sistema comprehensivo de todo lo recordado como presente a nuestra percepción interna o a nuestros sentidos. Llamamos realidad a cada individuo de ese sistema conectado con las impresiones presentes. Pero la mente no se detiene aquí. En efecto, al encontrar que junto a ese sistema de percepciones hay otro conectado por la costumbre o, si se quiere, por la relación causa y efecto, procede a examinar sus ideas; y como se da cuenta de que está en cierto modo necesariamente determinada a examinar estas ideas particulares y de que la costumbre o la relación -por la que está determinada- no admite el menor cambio, dispone estas ideas dentro de un nuevo sistema y las dignifica igualmente

26 Para Hume, una de las problemáticas fundamentales al momento de abordar el conocimiento es la ambigüedad y oscuridad de los términos: "El principal obstáculo, por consiguiente, para nuestro progreso en las ciencias morales o metafísicas es la oscuridad de las ideas y ambigüedad de los términos" (E.7.2, SO.153). 
con el título realidades. El primero de estos sistemas constituye el objeto de la memoria y de los sentidos; el segundo, del juicio (T.1.3.9.3 SB107-108, D.177) ${ }^{27}$.

Las ideas, por tanto, conforman sistemas orquestados bajo el principio de asociación, el cual puede dar lugar a la conformación de nuevas estructuras de conocimiento en un ritmo "variable" que dependerá tanto de la propia experiencia del sujeto como de la capacidad y habilidad de la imaginación. El conocimiento, por tanto, es un sistema "articulado por grados de fuerzas" que enlazan, separan, articulan o desarticulan impresiones e ideas que conforman la percepción y las cuales pueden ser plasmadas y analizadas desde las proposiciones. Hume, por consiguiente, asume y destaca la importancia del concepto de "atracción" que conduce a una interpretación dinámica y abierta del conocimiento, compuesta por distintos grados de fuerza y vivacidad, que representan nuestras impresiones (sensaciones, pasiones y emociones) e ideas (Santucci 1971, pp. 71,78), en palabras del autor:

En una palabra, la naturaleza ha proporcionado a ciertas impresiones e ideas una especie de atracción por la que, cuando una de ellas aparece, se presenta naturalmente su correlativa. Si estas dos atracciones o asociaciones de impresiones e ideas coinciden en el mismo objeto, se prestan ayuda mutua, con lo que la transición de las afecciones y la imaginación se produce con la mayor soltura y facilidad. Cuando una idea origina una impresión relacionada con otra impresión conectada, a su vez, con una idea relacionada con la idea primera, estas dos impresiones deben hacerse en cierto modo inseparables (...) (T.2.1.5.10 SB289, D.406).

En consecuencia, el entendimiento humano ${ }^{28}$, al cual concierne la operatoria de la memoria e imaginación en su conjunto, posee la asombrosa capacidad de articular y rearticular ideas en la forma de sistemas, los cuales en su generalidad constituyen el conocimiento.

Tercero, el conocimiento como sistema integrado de impresiones e ideas. El conocimiento como sistema recoge las impresiones sensibles en toda su complejidad, añadiendo al conocimiento de ideas el fundamental rol de las pasiones al momento de comprender nuestra experiencia. De este modo, el papel que juegan las pasiones en la concepción general del conocimiento para el filósofo de Edimburgo (Stroud 1986, p. 244) determina no solo la impronta y el carácter de las ideas en nuestra mente sino, además, el estado del sujeto frente a las mismas.

El esfuerzo de Hume y su particular desafío obedecen a la necesidad de abordar a la naturaleza humana en "toda su complejidad", éste es su particular desafío, posición que tiene una singular vigencia para nuestros tiempos y abre campo al análisis de distintas temáticas relativas al conocimiento y que se extiende incluso a problemas

27 Las cursivas introducidas son mías.

28 En este contexto, Hume reconoce la mente humana como colección o sistema de percepciones (T.1.3.9.3 SB107-108, D.177; Russell 2009, pp. 713-714; Stroud 1986, pp. 174 y ss.; Pereira 2014, pp. 209-210). 
éticos. La descripción en torno a la interacción entre impresiones e ideas es descrita por Hume desde su análisis del rol de las pasione ${ }^{29}$ y que tiene fundamental incidencia en la comprensión del principio ético que señala el bien en términos de "búsqueda del placer y rechazo del dolor" por el cual se realiza la construcción ética del sujeto (Stroud 1996, pp. 247-249), siempre con el propósito de alcanzar su bienestar, referente del análisis ético en el pensamiento empirista británico. En torno a estas temáticas podemos establecer la necesidad de una mirada unitaria entre impresiones e ideas al momento de analizar el conocimiento ${ }^{30}$, esto es, al reconocer que las emociones inciden (Stroud 1986, pp. 26, 233; Santucci 1971, pp. 88, 90-91) en nuestra visión del mundo (T.2.1.5.5 SB286, D.402-403). El estudio de todos estos aspectos cae bajo la consideración del concepto de conocimiento comprendido como sistema desde el cual, como he dicho, interactúan abiertamente pensamientos y emociones.

Es manifiesto, por tanto, que el papel de las impresiones en nuestro conocimiento se rige por las distintas sensaciones que se evocan en nuestra mente, generando distintos estados frente a nuestras ideas, que en ocasiones dependen del singular ritmo de nuestra propia experiencia. Sobre el particular, Hume en la Investigación sobre el Entendimiento Humano sostiene que un ejemplo en torno a las ideas que evocan emociones es el poema épico, en particular, cuando el poeta habla sobre los héroes:

Es evidente que en una composición correcta todas las afecciones excitadas por los diferentes eventos descritos y representados se añade recíprocamente fuerza; y que mientras los héroes entren todos en una escena común, y cada acción esté fuertemente conectada con el conjunto, el interés se mantendrá continuamente despierto y las pasiones fácilmente transitarán de una cosa a otra. La fuerte conexión de los eventos, dado que facilita el paso del pensamiento o de la imaginación de uno a otro, facilita también la transfusión de las pasiones, manteniendo las afecciones en el mismo cauce y en la misma dirección (E.3.12, SO.77).

Por consiguiente, la concepción del conocimiento como un sistema articula una estricta relación entre impresiones e ideas desde las cuales se despliega nuestra personal comprensión del mundo (T.2.1.5.6 SB287, D.403). Ahora bien, la complejidad de la

29 Un ejemplo de ello, según Hume: "Si examinamos ahora la mente humana, encontramos que, por lo que respecta a las pasiones, su naturaleza no es la de un instrumento musical a viento, en donde, una vez recorridas todas las notas, cesa inmediatamente el sonido en cuanto se deja de soplar, sino que se asemeja más bien a un instrumento de cuerda, en el que, después de cada golpe de arco, las vibraciones siguen reteniendo algún sonido, que va desvaneciéndose gradual e imperceptiblemente" (T. 2.3.9.12 SB440, D543; Santucci 1971, p. 76).

30 En torno a la materia moral, Stroud afirma: "Incluso en las esferas de la vida humana más aparentemente intelectuales o cognoscitivas, incluso en nuestros juicios empíricos sobre el mundo y en el proceso mismo del razonamiento puro, el sentimiento se muestra como la fuerza dominante (...) y la tarea de la ciencia consiste en descubrir empíricamente por qué tales pensamientos, creencias, sentimientos y acciones surgen del modo en que lo hacen" (Stroud 1986, p. 25). 
naturaleza humana en la que se enlazan pensamientos y emociones constituye el sendero desde el cual se nos devela nuestra forma de comprender los hechos, comprensión alejada del antiguo modo de entender el conocimiento, esto es, desde el mero examen de razonamientos abstractos: "La naturaleza siempre mantendrá sus derechos y prevalecerá sobre todo razonamiento abstracto" (E.5.1.2, SO117).

\section{Conclusiones}

El presente estudio nos conduce a reconocer las siguientes conclusiones: primero, el conocimiento según Hume mantiene el análisis de Berkeley en el cual destaca, por un lado, la crítica al concepto de sustancia como "algo desconocido" subyacente a las cosas, y por otro, la comprensión de las ideas abstractas en cuanto ideas expresadas en términos que comprenden conjuntos de ideas particulares. Segundo, el conocimiento se erige desde la conexión entre impresiones e ideas. Hume reconoce las ideas como copias débiles de las impresiones, en las cuales destaca su fuerza y vivacidad. Impresiones e ideas son enlazadas por el principio de asociación, el cual ata y desata en virtud de su atracción a cargo de las facultades de la memoria y la imaginación, posición que implica su distancia del concepto de sustancia e ideas abstractas como garantes del conocimiento objetivo. Tercero, el conocimiento consiste en un sistema. La estructura del conocimiento se articula bajo la unidad de un sistema por el principio de asociación el cual integra sensaciones, pasiones, sentimientos e ideas, los cuales en atención a la constancia y coherencia de las percepciones producen determinados hábitos en la mente que posibilitan la configuración de nuestro conocimiento por costumbres y creencias.

En el pensamiento de Hume cabe destacar la elaboración de una teoría del conocimiento distante de una concepción ontológica. Esta dimensión reconoce, por un lado, el central rol del análisis del lenguaje en la comprensión ideas, y por otro, el examen de la estructura psicológica del entendimiento marcada por la conexión entre impresiones e ideas. Cabe señalar, además, que si bien existen posiciones críticas en torno a la solidez de la epistemología de Hume (Russell 2009, p. 713; Noxon 1987, p. 134; Stroud 1986, pp. 326-328), es necesario reconocer el papel histórico del pensamiento del escocés al momento de reconocer los avances de la filosofía posterior.

Nuestro conocimiento es un sistema "articulado de fuerzas" en el cual se interconectan nuestras impresiones e ideas, sistema en el que nuestros recuerdos atesorados en la memoria y nuestros sueños elaborados por la imaginación posibilitan una lectura dinámica de los diversos eventos que podemos percibir. El filósofo de Edimburgo señala el necesario análisis del lenguaje en la comprensión del concepto de conocimiento, el cual se aleja del examen de sustancias por descubrir y posibilita la aproximación a experiencias que integran pensamientos y emociones. De esto modo, el estudio del conocimiento como sistema constituye un paso crucial que separa a Hume de la comprensión de ideas como meros razonamientos abstractos para adentrarse en el ingente desafío de la descripción de un mundo interior tan asombrosamente vasto y complejo como el de nuestras propias percepciones. La reflexión del pensador de Edimburgo intenta mostrar, por tanto, el flujo de esta "constelación de experiencias" 
que conforma nuestro conocimiento comprendido como una red que se entreteje como un sistema. En definitiva, podríamos interpretar, desde David Hume, que la pasión de nosotros, seres humanos, por nuestros conocimientos considera no solo la búsqueda de la solidez de nuestras afirmaciones, sino también la de nuestras profundas convicciones que se erigen desde nuestras íntimas costumbres y creencias. Convicciones que nos acompañan en esta singular sendero llamado "conocimiento".

\section{Referencias bibliográficas}

Ayer J. (1988), Hume. Trad. de J. C. Armero. Madrid: Alianza Editorial.

Berkeley G. (1980), Principios del conocimiento humano. Trad. de Pablo Masa. Buenos Aires: Losada.

(1967), The Principles of Human Knowledge, edited by G. J. Warnock. London: The Fontana Library.

Burlando G. (2013), Sobre el conocimiento y el significado en el Essay de John Locke, Veritas (29):119-137.

Gibson J. (1968), Locke's Theory of knowledge and its historical relations. London: Cambridge at the University Press.

Locke J. (1969), An Essay Human understanding (Albridge and edited by A.S. Pringle-Pattison). UK: Oxford at the Clarendon Press.

(1994), Ensayo sobre el entendimiento humano. Trad. de Edmundo O'Gorman. Colombia: FCE.

Hume D. (2000), A Treatise of Human Nature, edited by David Fate Norton And Mary J. Norton. UK: Oxford University Press.

(2011), Tratado de la naturaleza humana. Trad. de Félix Duque. Madrid: Ed. Tecnos.

(2004), Investigación sobre el entendimiento humano. Trad. de V. Sanféliz y C. Ors, Bilingüe. Madrid: Ed. Istmo.

Noxon J. (1987), La evolución de la filosofía de David Hume. Trad. de Carlos Solís. Madrid: Alianza Editorial.

Ockham G. (1986), Los sucesivos. Trad. de Francisco Fortuny. Barcelona: Ed. Orbis.

Pereira F. (2014), Hume y la ficción de la identidad personal, Ideas y valores 73 (154): 191-213.

Russell B. (1992), The basic writings of Bertrand Russell. London: Routledge.

(2009), Historia de la Filosofía. Trad. de Julio Gómez de la Serna y Antonio Dorta. Madrid: RBA.

Santucci A. (1971), Introduzione a Hume. Roma: Editori Laterza.

Stroud B. (1986), Hume. Trad. de Antonio Zirion. México: UNAM. 